\title{
MULTIPLIERS OF GROUP ALGEBRAS OF VECTOR-VALUED FUNCTIONS
}

\author{
U. B. TEWARI, M. DUTTA AND D. P. VAIDYA
}

\begin{abstract}
Let $G$ be a locally compact abelian group and $X$ be a Banach space. Let $L^{1}(G, X)$ be the Banach space of $X$-valued Bochner integrable functions on $G$. We prove that the space of bounded linear translation invariant operators of $L^{1}(G, X)$ can be identified with $L(X, M(G, X))$, the space of bounded linear operators from $X$ into $M(G, X)$ where $M(G, X)$ is the space of $X$-valued regular, Borel measures of bounded variation on $G$. Furthermore, if $A$ is a commutative semisimple Banach algebra with identity of norm 1 then $L^{1}(G, A)$ is a Banach algebra and we prove that the space of multipliers of $L^{1}(G, A)$ is isometrically isomorphic to $M(G, A)$. It also follows that if dimension of $A$ is greater than one then there exist translationinvariant operators of $L^{1}(G, A)$ which are not multipliers of $L^{1}(G, A)$.
\end{abstract}

1. Introduction. Let $G$ be a locally compact abelian group roup and let $\Gamma$ be the dual of $G$. Suppose $A$ is a commutative semisimple Banach algebra with an identity of norm one. The space $L^{1}(G, A)$ forms a commutative Banach algebra under convolution (see [2] and [5]). A bounded linear operator $T$ on $L^{1}(G, A)$ to itself is called a multiplier if $T$ commutes with convolutions, i.e. $T(f * g)=f * T g$ for all $f, g \in L^{1}(G, A)$. The set of all such operators is a closed subalgebra of the Banach algebra of all bounded linear operators on $L^{1}(G, A)$. This subalgebra is known as the multiplier algebra of $L^{1}(G, A)$. A bounded linear operator $T$ on $L^{1}(G, A)$ is said to be invariant if it commutes with translations. In the scalar-valued case, i.e. when $A=C$, the complex field, it is well known that a bounded linear operator $T$ on $L^{1}(G)$ (for $A=C$, we suppress the index ' $A$ ') is a multiplier if and only if it invariant. Moreover, the multiplier algebra in this case is isometrically isomorphic to $M(G)$, the space of bounded regular Borel measures on $G$ (see Theorem 0.1.1 of [6]). Akinyele claims in Theorem 3.2 of [7] that for an arbitrary $A$, a bounded linear operator $T$ on $L^{1}(G, A)$ is a multiplier if and only if $T$ is invariant. His claim is obviously false when $G$ is the trivial group consisting only of an identity element. In this case, $L^{1}(G, A)=A$ and all bounded operators on $A$ are trivially invariant. However, if dimension of $A$ is greater than 1 , there are bounded operators on $A$ which are not multipliers as can be seen from the proof of Theorem 3 of this paper. Actually, Theorem 3 shows that his claim is false for any locally compact abelian group.

In this paper, we prove that the space of bounded linear invariant operators on $L^{1}(G, X)$ can be identified with $L(X, M(G, X))$. We also prove that the space of

Received by the editors July 12, 1979.

AMS (MOS) subject classifications (1970). Primary 43A22.

Key words and phrases. Locally compact abelian group, invariant operators, multiplier. 
multipliers of $L^{1}(G, A)$ is isometrically isomorphic to $M(G, A)$. Finally, with the help of our results we clarify the status of various implications in Theorem 3.2 of [7].

2. Preliminaries. For integration of vector-valued set functions, we follow the developments as outlined in [4]. Using such integrals, it is possible to define convolutions between various spaces of vector-valued functions and measures on $G$. Indeed, under convolution, the spaces $L^{1}(G, X), M(G, X)$ and $C_{0}(G, X)$ become $L^{1}(G)$-Banach modules. Here, $C_{0}(G, X)$ denotes the Banach space of $X$-valued continuous functions on $G$, vanishing at infinity under the norm $\|F\| \|_{\infty}=$ $\sup _{x \in G}\|F(x)\|$, for $F \in C_{0}(G, X)$. The theory of vector measures and integration lets us identify the dual of $C_{0}(G, X)$ with $M\left(G, X^{*}\right)$, where $X^{*}$ is the dual of $X$ (see $\$ 19$ of [1] and [11]). The identification is given by $\langle\mu, F\rangle=\int F d \mu$, for $F \in$ $C_{0}(G, X)$ and $\mu \in M\left(G, X^{*}\right)$.

We shall make crucial use of the concept of module tensor products and their relations to multipliers (see [9] and [10]). If $V$ and $W$ are $A$-modules, the $A$-module tensor product $V \otimes_{A} W$ is defined to be the quotient Banach space $V \otimes_{\gamma} W / K$, where $K$ is the closed linear subspace of the projective tensor product $V \otimes_{\gamma} W$, spanned by the elements of the form $a v \otimes w-v \otimes a w$ with $a \in A, v \in V$ and $w \in W$. A continuous linear transformation from $V$ to $W$ is called an $A$-module homomorphism, if $T(a \circ v)=a \circ T(v)$ for all $a \in A$ and $v \in V$. The space of $A$-module homomorphisms from $V$ to $W$, denoted by $\operatorname{Hom}_{A}(V, W)$, is a Banach space under the operator norm. $\operatorname{Hom}_{A}(V, V)$ will be denoted by $\operatorname{Hom}_{A}(V)$. Moreover, we will write $\bigotimes_{G}$ for $\bigotimes_{L^{\prime}(G)}$, and $\operatorname{Hom}_{G}$ for $\operatorname{Hom}_{L^{\prime}(G)}$.

Rieffel has shown (2.12 and 2.13 of [9]), that there is a natural isometric isomorphism between $\operatorname{Hom}_{A}\left(V, W^{*}\right)$ and $\left(V \otimes_{A} W\right)^{*}$, under which an operator $T \in \operatorname{Hom}_{A}\left(V, W^{*}\right)$ defines a linear functional on $V \otimes_{A} W$ with value $\langle T(v), w\rangle$ at the element $v \otimes w$ of $V \otimes_{A} W$. If $A$ is a commutative, semisimple Banach algebra then a multiplier of $A$ is defined to be a bounded linear operator $T$ on $A$ such that $T(a b)=a T(b)$. We note that if $A$ is considered as a Banach module over itself then the multipliers of $A$ are nothing but the $A$-module homomorphisms of $A$.

The following lemmas will be useful subsequently.

LEMMA 1. There exists an isometric isomorphism between $L^{1}(G) \otimes_{G} C_{0}(G, X)$ and $C_{0}(G, X)$ which maps $f \otimes F$ into $f * F$, where $f \in L^{1}(G)$ and $F \in C_{0}(G, X)$.

Proof. The bilinear map $(f, F) \rightarrow f * F$ from $L^{1}(G) \times C_{0}(G, X)$ into $C_{0}(G, X)$ gives rise to a bounded linear map of norm $<1$ from $L^{1}(G) \otimes_{G} C_{0}(G, X)$ into $C_{0}(G, X)$. Since $L^{1}(G)$ contains a bounded approximate identity, we can use the arguments of (3.3) of [10] to show that this map is injective. It can be easily seen that the set $\left\{f * F: f \in L^{1}(G)\right.$ and $\left.F \in C_{0}(G, X)\right\}$ is dense in $C_{0}(G, X)$. Now, it is immediate from Theorem 32.22 of [3] that the above map is surjective. For the isometry, we observe that $d=1$ in the present context, and then parts (ii) and (iv) of Theorem 32.22 together imply that for any $D>d$ there is a factorization $y=a z$ with $\|a\|\|z\| \leqslant D\|y\|$. 
Lemma 2. Let $f \in L^{1}(G), F \in C_{0}(G, X)$ and $\mu \in M\left(G, X^{*}\right)$. Then

$$
\langle\mu * f, F\rangle=\langle\mu, f * F\rangle \text {. }
$$

Proof. A simple application of the Fubini theorem gives the result for the scalar-valued case. Using this and some simple properties of the vector-valued integral, the lemma is easily proved for any $F$ of the form $F=\sum_{i=1}^{n} g_{i} x_{i}$ with each $g_{i} \in C_{0}(G)$ and $x_{i} \in X$. Since functions of this form are dense in $C_{0}(G, X)$, the result is true for any $F \in C_{0}(G, X)$ and the lemma is proved.

It is well known that the convolution of two elements $f, g \in L^{1}(G)$ can be expressed as an $L^{1}(G)$-valued integral (see $\S 6.6$ of Chapter 3 of [8]). A similar result can be obtained for $f \in L^{1}(G, X)$ and $g \in L^{1}(G)$. Using this we can easily prove the following two lemmas.

LEMMA 3. Let $T$ be an invariant bounded linear operator from $L^{1}(G)$ into $L^{1}(G, X)$. Then $T \in \operatorname{Hom}_{G}\left(L^{1}(G), L^{1}(G, X)\right)$, i.e. $T(f * g)=f * T(g)$ for $f, g \in$ $L^{1}(G)$.

LEMma 4. Let $T$ be an invariant bounded linear operator of $L^{1}(G, X)$. Then $T \in \operatorname{Hom}_{G}\left(L^{1}(G, X)\right)$.

3. The space $\operatorname{Hom}_{G}\left(L^{1}(G), L^{1}(G, X)\right)$. We shall now prove that $\operatorname{Hom}_{G}\left(L^{1}(G), L^{1}(G, X)\right)$ is isometrically isomorphic to $M(G, X)$. If $f \in L^{1}(G)$ and $\mu \in M(G, X)$, then considering $f$ as an element of $M(G), \mu * f$ is defined as an element of $M(G, X)$. This is because $(X, C, X)$ forms a bilinear system in the sense of Chapter II of [4] and hence we can use the results of Chapter IV of [4] to define $\mu * f$. It is not very difficult to prove that $\mu * f$, so defined, actually belongs to $L^{1}(G, X)$. Indeed, if $f$ is a continuous function having compact support then $\mu * f$ has the following integral representation.

$$
\mu * f(s)=\int_{G} f\left(t^{-1} s\right) d \mu(t)
$$

Also, it follows from the usual properties of convolutions, that $f \rightarrow \mu * f$ is a bounded linear invariant operator from $L^{1}(G)$ into $L^{1}(G, X)$. By Lemma 3 , this defines an element of $\operatorname{Hom}_{G}\left(L^{1}(G), L^{1}(G, X)\right)$. We now prove

Theorem 1. Let $T \in \operatorname{Hom}_{G}\left(L^{1}(G), L^{1}(G, X)\right)$. Then there exists a $\mu \in M(G, X)$ such that $T(f)=\mu * f$ for every $f \in L^{1}(G)$. Moreover, this correspondence gives an isometric isomorphism between $\operatorname{Hom}_{G}\left(L^{1}(G), L^{1}(G, X)\right)$ and $M(G, X)$.

Proof. Let $T \in \operatorname{Hom}_{G}\left(L^{1}(G), L^{1}(G, X)\right)$. Considering the natural isometric embedding of $X$ in its second dual $X^{* *}, L^{1}(G, X)$ can be embedded isometrically in $M\left(G, X^{* *}\right)$. Hence $T$ can be considered as an element of $\operatorname{Hom}_{G}\left(L^{1}(G), M\left(G, X^{* *}\right)\right)$ with the same norm. However, $M\left(G, X^{* *}\right)$ is isometrically isomorphic to $C_{0}\left(G, X^{*}\right)^{*}$. Hence, by Rieffel's result on module homomorphisms (see §2), there exists $\varphi \in\left(L^{1}(G) \otimes_{G} C_{0}\left(G, X^{*}\right)\right)^{*}$, with $\|\varphi\|=\|T\|$, such that $\langle\varphi, f \otimes F\rangle=\langle T(f), F\rangle$ for $F \in C_{0}\left(G, X^{*}\right)$ and $f \in L^{1}(G)$. Here, $T(f)$ has been considered as an element of $M\left(G, X^{* *}\right)$ which is the dual of $C_{0}\left(G, X^{*}\right)$. However, by Lemma $1, L^{1}(G) \otimes_{G} C_{0}\left(G, X^{*}\right)$ is isometrically isomorphic to 
$C_{0}\left(G, X^{*}\right)$ under the map $f \otimes F \rightarrow f * F$. Hence, there exists $\mu \in M\left(G, X^{* *}\right)$ with $\|\mu\|=\|T\|$ such that $\langle\mu, f * F\rangle=\langle T(f), F\rangle$ for $F \in C_{0}\left(G, X^{*}\right)$ and $f \in L^{1}(G)$. Hence, by Lemma 2, we have $\langle T(f), F\rangle=\langle\mu * f, F\rangle$, for every $F \in C_{0}\left(G, X^{*}\right)$. Therefore $T(f)=\mu * f$ for every $f \in L^{1}(G)$. The association of $\mu$ with $T$ is obviously linear and since $\|\mu\|=\|T\|$, the proof of the theorem will be complete if we prove that $\mu$ takes values only in $X$.

Let us take the canonical map $\psi$ from $X^{* *}$ onto the quotient space $Y=X^{* *} / X$. Consider the $Y$-valued measure $\nu=\psi \circ \mu$. Obviously $\nu \in M(G, Y)$ and $\nu * f=$ $\psi \circ(\mu * f)$, for any $f \in L^{1}(G)$. However $\mu * f=T(f) \in L^{1}(G, X)$ and hence, for any measureable set $E, \mu * f(E) \in X$. This means that $\nu * f=0$ for any $f \in$ $L^{1}(G)$. Consider $\nu$ as an element of $M\left(G, Y^{* *}\right)$ which is the dual of $C_{0}\left(G, Y^{*}\right)$. Take any $F \in C_{0}\left(G, Y^{*}\right)$. Then $F=f * F^{\prime}$ for some $f \in L^{1}(G)$ and $F^{\prime} \in$ $C_{0}\left(G, Y^{*}\right)$ (see the proof of Lemma 1). Therefore,

$$
\langle\nu, F\rangle=\left\langle\nu, f * F^{\prime}\right\rangle=\left\langle\nu * f, F^{\prime}\right\rangle=0 .
$$

Hence $\nu=0$, i.e. $\psi(\mu(E))=0$ for any measurable set $E$. This shows that $\mu(E) \in X$ for any measurable set $E$ and our proof is complete.

Corollary. $\operatorname{Hom}_{G}\left(L^{1}(G), L^{1}(G, X)\right)$ is precisely the set of invariant bounded linear operators from $L^{1}(G)$ into $L^{1}(G, X)$.

4. Invariant operators of $L^{1}(G, X)$. We now characterise the invariant bounded linear operators of $L^{1}(G, X)$. Let $T$ be such an operator. Then, by Lemma 4, $T \in \operatorname{Hom}_{G}\left(L^{1}(G, X)\right)$. Take any $x \in X$, and define a mapping $T_{x}$ from $L^{1}(G)$ into $L^{1}(G, X)$ by $T_{x}(f)=T(f x)$. It is easy to prove that $T_{x} \in \operatorname{Hom}_{G}\left(L^{1}(G), L^{1}(G, X)\right)$. Also $\left\|T_{x}\right\| \leqslant\|x\|\|T\|$. Therefore, by Theorem 1 , there exists a measure $M(x) \in$ $M(G, X)$ such that $T_{x}(f)=M(x) * f$ and $\|M(x)\|<\|x\|\|T\|$. It is easy to see that $x \rightarrow M(x)$ is linear and hence from $T$ we have obtained a bounded linear operator $M$ from $X$ into $M(G, X)$ with $\|M\| \leqslant\|T\|$ such that $T(f x)=M(x) * f$, for all $x \in X$ and $f \in L^{1}(G)$.

Conversely, let $M$ be a bounded linear operator from $X$ into $M(G, X)$. Then $(f, x) \rightarrow M(x) * f$ gives a bilinear map from $L^{1}(G) \times X$ into $L^{1}(G, X)$ such that $\|M(x) * f\|_{1}=\|M(x) * f\| \leqslant\|M(x)\|\|f\|_{1}<\|M\|\|x\|\|f\|_{1}$. Hence, by the universal property of tensor products with respect to bounded bilinear maps, we get a bounded linear map $T^{\prime}$ from $L^{1}(G) \otimes_{\gamma} X$ into $L^{1}(G, X)$ with $\left\|T^{\prime}\right\|<\|M\|$, such that $T \rightarrow(f \otimes x)=M(x) * f$ for any $f \in L^{1}(G)$ and $x \in X$. However, $L^{1}(G)$ $\otimes_{\gamma} X$ is isometrically isomorphic to $L^{1}(G, X)$. Hence we get a bounded linear operator $T$ of $L^{1}(G, X)$ with $\|T\| \leqslant\|M\|$, such that $T(f x)=M(x) * f$ for any $f \in L^{1}(G)$ and $x \in X$. Let $s \in G$. Then $T\left((f x)_{s}\right)=T\left(f_{s} x\right)=M(x) * f_{s}=$ $(M(x) * f)_{s}=(T(f x))_{s}$. Since functions of the form $\sum_{i=1}^{n} f_{i} x_{i}$ with $f_{i} \in L^{1}(G)$ and $x_{i} \in X$, are dense in $L^{1}(G, X)$, and since translation is continuous in $L^{1}(G, X)$, it follows that $T$ is invariant. By the previous paragraph, we can obtain a bounded linear transformation from $X$ into $M(G, X)$, associated with $T$. It is easy to see that this transformation is nothing but $M$, and hence $\|M\|<\|T\|$. Therefore $\|T\|=$ $\|M\|$. It is easy to see that this association is linear. Thus we have proved the following. 
TheOREM 2. $\operatorname{Hom}_{G}\left(L^{1}(G, X)\right)$ is precisely the set of bounded linear invariant operators of $L^{1}(G, X)$ and this space is isometrically isomorphic to $L(X, M(G, X))$, the space of bounded linear operators from $X$ into $M(G, X)$.

5. Multipliers of $L^{1}(G, A)$. Let $A$ be a commutative, semisimple Banach algebra with an identity $e$ of norm one. For any $F \in L^{1}(G, A)$ we can define an $A$-valued function $\hat{F}$ on $\Gamma$ as follows.

$$
\hat{F}(\gamma)=\int_{G} \bar{\gamma}(s) F(s) d s .
$$

It is easy to prove that $\hat{F}$ is a bounded continuous function from $\Gamma$ into $A$. Also, for any $a \in A$ and $f \in L^{1}(G)$, we have $(a f)^{\hat{n}}=a \hat{f}$, where $\hat{f}$ is the usual Fourier transform. Using the fact that $A$ has an identity, it is easy to prove the following.

Lemma 5. Let $T$ be a multiplier of $L^{1}(G, A)$. Then there exists a unique continuous bounded function $\hat{T}$ on $\Gamma$ to $A$ such that for any $\gamma \in \Gamma,(T F)^{\wedge}(\gamma)=\hat{T}(\gamma) \hat{F}(\gamma)$ for every $F \in L^{1}(G, A)$.

COROllary 5.1. If $T$ is a multiplier of $L^{1}(G, A)$ then $T$ is invariant.

Corollary 5.2. If $T$ is a multiplier of $L^{1}(G, A)$, then $T(a F)=a T(F)$ for all $a \in A$ and $F \in L^{1}(G, A)$, i.e. $T \in \operatorname{Hom}_{A}\left(L^{1}(G, A)\right)$.

We are now in a position to disprove Akinyele's result mentioned in the introduction.

THEOREM 3. If dimension of $A>1$, then there exists a bounded linear invariant operator $T$ of $L^{1}(G, A)$ such that $T \notin \operatorname{Hom}_{A}\left(L^{1}(G, A)\right)$.

Proof. Let $\psi$ be a nonzero multiplicative linear functional on $A$. Define $\phi(a)=\psi(a) e$ for $a \in A$. Clearly $\phi$ is a bounded linear operator on $A$. From the fact that dimension of $A>1$, it is easy to see that for some $a \in A, \phi(a) \neq a \phi(e)$. Let $\delta$ be the Dirac measure with unit mass at the identity of $G$. Then $M(a)=\phi(a) \delta$ gives a bounded linear operator from $A$ into $M(G, A)$. By Theorem 2 there exists a bounded linear invariant operator $T$ of $L^{1}(G, A)$ such that $T(b f)=M(b) * f=$ $\phi(b) \delta * f=\phi(b) f$ for any $b \in A$ and $f \in L^{1}(G)$. Now since $\phi(a e) \neq a \phi(e)$, we have $T(a f)=\phi(a) f \neq a \phi(e) f=a T(e f)$. This shows that $T \notin \operatorname{Hom}_{A}\left(L^{1}(G, A)\right)$, which proves the theorem.

Since by Corollary 5.2 any multiplier of $L^{1}(G, A)$ belongs to $\operatorname{Hom}_{A}\left(L^{1}(G, A)\right)$, the above construction gives a bounded linear invariant operator $T$ of $L^{1}(G, A)$ which is not a multiplier of $L^{1}(G, A)$. This disproves Akinyele's result.

We now characterise the multipliers of $L^{1}(G, A)$. Corollary 5.2 shows that in order to be a multiplier an invariant operator $T$ of $L^{1}(G, A)$ must satisfy $T \in$ $\operatorname{Hom}_{A}\left(L^{1}(G, A)\right)$. It is easy to see that the condition is also sufficient. Now, let $\mu \in M(G, A)$ and $F \in L^{1}(G, A)$. Considering $F$ as an element of $M(G, A)$ we can use the results of Chapter IV of [4] to define $\mu * F$ as an element of $M(G, A)$. This is because $(A, A, A)$ forms a bilinear system in the sense of Chapter II of [4]. If $F$ is of the form $F=a f$ for $a \in A$ and $f \in L^{1}(G)$, then it is easy to see that $\mu * F \in$ $L^{1}(G, A)$ (just like the case of $\mu * f$; see the beginning of $\S 3$ ). Since linear 
combinations of functions of this form are dense in $L^{1}(G, A), \mu * F \in L^{1}(G, A)$ for all $F \in L^{1}(G, A)$. Using the properties of convolutions, it is easy to see that the operator $T$ of $L^{1}(G, A)$ defined by $T(F)=\mu * F$ is invariant and belongs to $\operatorname{Hom}_{A}\left(L^{1}(G, A)\right)$. Thus $T$ is a multiplier of $L^{1}(G, A)$. In the following theorem, we prove that all the multipliers of $L^{1}(G, A)$ are given by elements of $M(G, A)$ in this way.

Theorem 4. Let $T$ be a multiplier of $L^{1}(G, A)$. Then there exists a $\mu \in M(G, A)$ such that $T(F)=\mu * F$ for all $F \in L^{1}(G, A)$. Moreover, this correspondence gives an isometric isomorphism between $\operatorname{Hom}_{L^{\prime}(G, A)}\left(L^{1}(G, A)\right)$ and $M(G, A)$.

Proof. Let $T$ be a multiplier of $L^{1}(G, A)$. Then by Corollary 5.1 and Lemma 4, $T \in \operatorname{Hom}_{G}\left(L^{1}(G, A)\right)$. Therefore, by Theorem 2, there exists $M \in L(A, M(G, A))$ with $\|M\|=\|T\|$ and $T(f a)=M(a) * f$ for any $a \in A$ and $f \in L^{1}(G)$. By Corollary 5.2, $T \in \operatorname{Hom}_{A}\left(L^{1}(G, A)\right)$. Therefore,

$$
T(f a)=T(a(f e))=a T(f e)=a(M(e) * f)=M(e) *(f a) .
$$

Since linear combinations of functions of the form ' $f a$ ' are dense in $L^{\prime}(G, A)$, we have $T(F)=\mu * F$ for every $F \in L^{1}(G, A)$, where $\mu=M(e)$. Now, $\|\mu\|<$ $\|M\|\|e\|=\|T\|$. On the other hand, $\|T(F)\|_{1}<\|\mu\|\|F\|_{1}$. Therefore $\|T\|<$ $\|\mu\|$. This proves that $\|\mu\|=\|T\|$. It is obvious that the mapping $T \rightarrow \mu$ is linear. This completes the proof.

6. Remark on Akinyele's results. In this section, we shall examine the status of various implications in Theorem 3.2 of [7]. It will be helpful to state his theorem in our notation.

THEOREM A. Let $G$ be a locally compact abelian group and $A$ be a commutative semisimple Banach algebra. Suppose $T: L^{1}(G, A) \rightarrow L^{1}(G, A)$ is a bounded linear operator. Then the following are equivalent.

(i) $T$ is a multiplier of $L^{1}(G, A)$.

(ii) $T$ is invariant.

(iii) There exists a unique $m \in M(G, L(A))$ such that $T f=m * f$ for each $f \in$ $L^{1}(G, A) .(L(A)$ denotes the Banach algebra of all bounded linear operators on $A$.)

It can be easily seen that the implications (i) $\Rightarrow$ (ii), (i) $\Rightarrow$ (iii) and (iii) $\Rightarrow$ (ii) are true. We shall show that all other implications in Theorem $\mathbf{A}$ are false.

Theorem 3 shows that the implication (ii) $\Rightarrow$ (i) is false. In the notation of Theorem 3, if we consider the measure $\delta \cdot \varphi \in M(G, L(A))$, then the corresponding operator on $L^{1}(G, A)$ is not a multiplier, showing that the implication (iii) $\Rightarrow(i)$ is also false. To show that the implication (ii) $\Rightarrow$ (iii) is false, we consider the following example.

ExAmpLe. Let $G$ be the real line with the usual topology and let $A$ be the group algebra of the real line with the discrete topology. For $x \in A$, consider the set function $M(x)$ defined by $M(x)(B)=\chi_{B} \cdot x$, for every Borel subset $B$ of $G\left(\chi_{B}\right.$ denotes the characteristic function of $B)$. It can be easily seen that the function $M$ which associates $M(x)$ to each $x$ in $A$ is an element of $L(A, M(G, A))$. The 
operator on $L^{1}(G, A)$ corresponding to $M$ as in Theorem 2, satisfies condition (ii) but does not satisfy condition (iii) of Theorem A. This can be easily seen from the fact that the set function $m$ defined on the Borel subsets of $G$ by $m(B)(x)=$ $M(x)(B)$ takes values in $L(A)$, but $m$ is neither regular nor of bounded variation.

The other main theorem in Akinyele's paper is Theorem 3.1. The proof of this theorem, given in [7], appears to be wrong. The use of Theorem 2 in $\$ 19$ of [1] does not seem to be justified. It would be interesting to characterise the set of all bounded linear invariant operators on $C_{0}(G, A)$. Our methods do not appear to give such a characterisation.

ACKNOWLEDGement. The authors wish to express their sincere thanks to the referee for his helpful suggestions.

\section{REFERENCES}

1. N. Dinculeanu, Vector measures, Pergamon, Oxford, 1967.

2. A. Hausner, Group algebras of vector valued functions Bull. Amer. Math. Soc. 62 (1956), 383.

3. E. Hewitt and K. A. Ross, Abstract harmonic analysis, Vol. II; Structure and analysis for compact groups. Analysis on locally compact Abelian groups, Die Grundlehren der math. Wissenschaften, Band 152, Springer-Verlag, Berlin and New York, 1970.

4. J. E. Huneycutt, Jr., Products and convolutions of vector valued set functions, Studia Math. 41 (1972), $101-129$.

5. G. P. Johnson, Spaces of functions with values in a Banach algebra, Trans. Amer. Math. Soc. 92 (1959), 411-429.

6. R. Larsen, An introduction to the theory of multipliers, Springer-Verlag, Berlin and New York, 1971.

7. O. Akinyele, A multiplier problem, Atti Accad. Naz. Lincei Rend. Cl. Sci. Fis. Mat. Natur. (8) 57 (1974), no. 6, 487-490 (1975).

8. H. Reiter, Classical harmonic analysis and locally compact groups, Oxford Univ. Press, Oxford, 1968.

9. M. Rieffel, Induced Banach representations of Banach algebras and locally compact groups, J. Functional Analysis 1 (1967), 443-491.

10. $\quad$ Multipliers and tensor products of $L^{P}$-spaces of locally compact groups, Studia Math. 33 (1969), 71-82.

11. I. Singer, Linear functionals on the space of continuous mappings of a compact Hausdorff space into a Banach space, Rev. Math. Pures. Appl. 2 (1957), 301-315.

DePartment of Mathematics, Indin Institute of Technology, KANPUR, 208016, INDIA 